\title{
Examining the validity of Stoney-equation for in-situ stress measurements in thin film electrodes using a large-deformation finite-element procedure
}

\author{
Jici Wen ${ }^{\mathrm{a}, \mathrm{c}}$, Yujie Wei ${ }^{\mathrm{a}, \mathrm{c}, *}$, Yang-Tse Cheng ${ }^{\mathrm{b}}$ \\ a State Key Laboratory of Nonlinear Mechanics (LNM), Institute of Mechanics, Chinese Academy of Sciences, Beijing 100190, Chine \\ b Department of Chemical and Materials Engineering, University of Kentucky, Lexington, KY 40506, USA \\ ${ }^{\mathrm{c}}$ School of Engineering Science, University of Chinese Academy of Science, Beijing 100049, China
}

\section{H I G H L I G H T S}

- Critical examination on the validity of Stoney equation in film electrodes.

- Effects of concentration dependent elasticity to in-situ stress measurements.

- Effects of finite deformation to in-situ stress measurements.

- A robust electrochemical-mechanical coupling FE procedure.

\section{A R T I C L E I N F O}

\section{Keywords:}

Thin film electrode

In-situ stress measurement

Stoney equation

Diffusion-stress coupling

Finite-deformation finite element

\begin{abstract}
A B S T R A C T
During the lithiation and delithiation of a thin film electrode, stress in the electrode is deduced from the curvature change of the film using the Stoney equation. The accuracy of such a measurement is conditioned on the assumptions that (a) the mechanical properties of the electrode remain unchanged during lithiation and (b) small deformation holds. Here, we demonstrate that the change in elastic properties can influence the measurement of the stress in thin film electrodes. We consider the coupling between diffusion and deformation during lithiation and delithiation of thin film electrodes and implement the constitutive behavior in a finitedeformation finite element procedure. We demonstrate that both the variation in elastic properties in thin film electrodes and finite-deformation during lithiation and delithiation would challenge the applicability of the Stoney-equation for in-situ stress measurements of thin film electrodes.
\end{abstract}

\section{Introduction}

Battery performance is largely compromised by the stress in active cathode and anode particles because of large volume change associated with the diffusion of lithium-ions during battery charge/discharge cycles [1-3]. Understanding battery degradation has stimulated a growing interest in studying the electrochemical-mechanical coupled fields and the subsequent stress variation during lithiation and delithiation. Many researchers are focusing on Si-based and Sn-based anodes because of their high charge capacities and relatively low density. However, high capacities usually are associated with huge volume expansion and high stress, causing poor cycle life. A robust numerical implementation of diffusion-finite deformation in strongly coupled phenomena is, therefore, necessary for simulating large strain $(\sim 300 \%)$ in silicon- or tin-based electrodes for lithium ion battery applications.
It is generally agreed that the lithiation and delithiation in batteries involve electrochemical-mechanical coupling. Taking $\mathrm{Si}$ as an example, the electrochemical lithiation of crystalline Si exhibits complicated structural changes [4], such as phase transition and volume changes [5]. As a result, significant stress change occurs, which has been related to the performance of the battery. Given the experimental difficulties in quantifying the coupling effects, intensive endeavors have been made in developing numerical methods in the past decade to capture the electrochemical-mechanical processes in batteries [6-12]. Anand et al. (2012) [13] has used a Cahn-Hilliard-type theory for species diffusion coupled with large elastic-plastic deformations.

When applying the Stoney equation for stress measurements $[14,15]$, the accuracy is contingent upon the assumption that the elastic and plastic properties of the electrode remain unchanged during lithiation. However, there are obvious changes in the mechanical properties in the process of lithiation and delithiation of electrodes [16-18].

\footnotetext{
* Corresponding author. State Key Laboratory of Nonlinear Mechanics (LNM), Institute of Mechanics, Chinese Academy of Sciences, Beijing 100190, China.

E-mail address: yujie_wei@lnm.imech.ac.cn (Y. Wei).
} 
In this paper, we demonstrate via finite element method based computations how the variation of elastic properties could influence the stress measurement and the stress-capacity relationship in thin film battery electrodes. We first present in Sec. 2 the numerical implementation of diffusion-finite deformation strongly coupled problems. We report the curvature response as a function of lithium concentration-dependent elastic modulus and discuss the limitation of the Stoney equation for stress measurements in thin film electrodes in Sec. 3. We conclude in Sec. 4 with final remarks.

\section{The finite element formula}

Here we introduce the diffusion-induced finite deformation coupled equations for modeling the electrode's finite deformation in the process of charge or discharge. Previously, many researchers have demonstrated that there exists a stress-capacity hysteresis in electrode materials, and the irreversible deformation is greater than the elastic deformation [17-20]. We introduce, therefore, a large inelastic deformation theory to our diffusion-finite deformation fully coupled theory, which, we believe, can be applied to many electrode materials. We discuss, in the following, diffusion process and finite deformation process separately.

\subsection{Diffusion process for the finite deformation}

At any given time $t$, the lithium ion concentration $\phi(x, t)$ at a material point $\boldsymbol{x}$ in the electrode structure (v) is governed by the diffusion equation [21].

$\frac{\partial \phi}{\partial t}+\nabla \cdot \boldsymbol{J}=0($ in $v)$

where $\nabla=\frac{\partial}{\partial x_{i}}$ is the gradient relative to current position $x_{i}$ [22]. Hence the initial configuration $\boldsymbol{X}$ coincides with the current one $\boldsymbol{x}$ at $t=0$, i.e., $X_{i}=x_{i}(t=0)$, and the subscript $i$ runs from $x$ to $z$ in the Cartesian coordinate system. The boundary condition is given as

$\phi=\widetilde{\phi},\left(\right.$ on $\left.\Gamma_{1}\right)$ and $\boldsymbol{J} \cdot \boldsymbol{n}=J_{i} n_{i}=-J^{s}\left(\right.$ on $\left.\Gamma_{2}\right)$

Here $J^{s}$ is the component of surface flux, $\boldsymbol{n}$ is the surface normal, and $\Gamma_{1}$ and $\Gamma_{2}$ represent the surface of the volume $v$. Here, the galvanostatic current boundary condition $J^{s}$ is related to the current density $i^{s}$ via $J^{s}=\frac{i^{s}}{F}$ [23], and $F$ is the Faraday constant.

There are several models for the effect of stress on lithium ion flow. The commonly used one expresses the flux, $\boldsymbol{J}$, a function of the gradient of a stress-dependent chemical potential $\mu$ (e.g. [13,22,24-27])

$\boldsymbol{J}=-\frac{D(\phi, \boldsymbol{x})}{R T} \nabla \mu\left(\phi, \sigma_{h}, \boldsymbol{x}, t\right)$

where $D(\phi, \boldsymbol{x})$ is the diffusivity depending on concentrations and current positions, $R$ is the gas constant and $T$ is the absolute temperature. Note that different groups may use slightly different formula of $\mu$ although the same factors were taken into account (e.g. [13,22,27]). Eqn. (1c) reflects the influence of stress on concentration and vice versa. As the exact dependence of $\mu$ on $\sigma$ remains debatable, we adopt a simple form (e.g. [24-27])

$\mu\left(\phi, \sigma_{h}, \boldsymbol{x}, t\right)=\mu_{0}+R \ln \frac{\phi}{\phi_{\max }}-\Omega \sigma_{h}$

where is the referential potential, $\phi_{\max }$ is the maximum lithium concentration, $\Omega$ is the lithium ions partial molar volume and $\sigma_{h}$ is the hydrostatic stress, i.e., $\left(\sigma_{x x}+\sigma_{y y}+\sigma_{z z}\right) / 3$. The second term represents an entropic contribution to the free-energy, while the third term is the work done by the applied stress. Combining Eqns. (1c) and (1d), the flux equation can be rewritten as
$\boldsymbol{J}=-\frac{D(\phi, \boldsymbol{x})}{\phi} \nabla \phi(\boldsymbol{x}, t)+\frac{D(\phi, \boldsymbol{x}) \Omega}{R T} \nabla \sigma_{h}$

We consider a linear dependence of diffusivity on concentration and consider isotropic diffusion $D(\phi, \boldsymbol{x})=D_{0} \phi$.

In general, the concentration $\phi$ is associated with compound phase $A_{\psi} B$ in the electrode where $A$ and $B$ indicate the lithium and the anode electrode material respectively, and the subscript $\psi$ indicates the lithium fraction ranging from 0 to $\psi_{\max }$. Its connection with $\phi$ is defined by

$\phi=\frac{\psi}{V_{\psi} N_{a}}$,

where $V_{\psi}$ is the volume of the compound $A_{\psi} B$ and is a function of $\psi$, and $N_{a}$ is Avogadro's number. Taking lithium-silicon battery system as an example, the volume of the compound $\mathrm{Li}_{\psi} \mathrm{Si}$ is $[28,29]$.

$V_{\psi}=\left(1+3.2 \frac{\psi}{\psi_{\max }}\right) \times 10^{-28} \mathrm{~m}^{3}$,

and the maximum lithium fraction is $\psi_{\max }=4.4\left(\mathrm{Li}_{4.4} \mathrm{Si}\right.$ [30]). For numerical convenience, we consider $\psi$ as a continuous variable. We write the diffusion equation in terms of $\psi$, and Eqn. (1a) is given as

$\frac{V_{\psi}-\psi V_{\psi}^{\prime}}{V_{\psi}^{2} N_{a}} \frac{\partial \psi}{\partial t}+\nabla \cdot \boldsymbol{J}=0($ in $v)$

where $V_{\psi}^{\prime}=\frac{\partial V_{\psi}}{\partial \psi}$, and the boundary conditions are

$\psi=\widetilde{\psi},\left(\right.$ on $\left.\Gamma_{1}\right)$ and $J_{i} n_{i}=-J^{s}\left(\right.$ on $\left.\Gamma_{2}\right)$

for prescribed concentration and ion flux, respectively. The flux equation (Eqn. (2)) is then written as

$\boldsymbol{J}=-D_{0} \nabla\left(\frac{\psi}{V_{\psi} N_{a}}\right)+\frac{D_{0} \Omega}{R T}\left(\frac{\psi}{V_{\psi} N_{a}}\right) \nabla \sigma_{h}$

According to eqn. (5a) and eqn. (5b), the variational form $\delta W_{d}$ of the above diffusion equation is given as

$\delta W_{d}=\int_{v} \delta \psi \frac{V_{\psi}-\psi V_{\psi}^{\prime}}{V_{\psi}^{2} N_{a}} \frac{\partial \psi}{\partial t} d v-\int_{v} \delta \psi_{, i} J_{i} d v-\int_{s} J^{s} \delta \psi d s=0$

where $v$ and $s$ are the volume and the surface of the deformed body, respectively. The differentiation of $\delta W_{d}$ is

$$
\begin{aligned}
d \delta W_{d}= & \int_{v} \delta \psi H(\psi) \frac{\partial d \psi}{\partial t} d v+\int_{v} \delta \psi_{, i} D_{0} H(\psi) d \psi_{, i} d v \\
& -\int_{v} \delta \psi_{, i} \frac{D_{0} \Omega}{R T} \sigma_{h, i} H(\psi) d \psi d v \\
& -\int_{v}\left[\delta \psi_{, i} G_{, m}(\psi) d u_{m, i}+\delta \psi_{, m} G_{, i}(\psi) d u_{m, i}\right] d v
\end{aligned}
$$

where $H(\psi)=\frac{\partial \phi}{\partial \psi}=\frac{V_{\psi}-\psi V_{\psi}^{\prime}}{V_{\psi}^{2} N_{a}}, G_{, i}(\psi)=D_{0} H(\psi) \psi_{, i}-\frac{D_{0} \Omega}{R T} \frac{\psi}{V_{\psi} N_{a}} \sigma_{h, i}$. The first three terms on the RHS of eqn. (7) are associated with diffusion, and the last one is the finite deformation-induced stiffness matrixes.

\subsection{The finite deformation problems for electrodes}

Since large volume changes occur in the negative electrode in high capacity batteries, and the Cauchy stress is affected by rigid-body rotation (not frame invariant), an objective stress rate with respect to the logarithm strain rate is desired. The objective stress rate can be acquired by the Jaumann rate [31] as

$\dot{\sigma}_{i j}{ }^{J}=\sigma_{i j}+\sigma_{i k} W_{k j}-W_{i k} \sigma_{k j}$ 
where $\sigma_{i j}{ }^{J}$ is the Jaumann stress rate; $\sigma_{i j}$ is the time rate of Cauchy stress, $W_{i j}=\left(L_{i j}-L_{j i}\right) / 2$ is the spin tensor, $L_{i j}=\frac{\partial v_{i}}{\partial x_{j}}=\dot{F} F^{-1}$ is the velocity gradient, and $F_{i j}=\frac{\partial x_{i}}{\partial X_{j}}$ is the deformation gradient tensor. The Jaumann stress rate can then be used to objectively describe the stress and strain rate $\left(\dot{\varepsilon}_{k l}\right)$ relation, $\dot{\sigma}_{i j}{ }^{J}=C_{i j k l} \dot{\varepsilon}_{k l}$ with $C_{i j k l}$ the fourth-order elastic modulus tensor. The rate-independent elastic-plastic constitutive relationship (coupled with diffusion) in incremental form is then given as

$d \sigma_{i j}^{J}=C_{i j k l}\left(d \varepsilon_{k l}^{m}-d \varepsilon_{k l}^{p l}\right)+\frac{\partial \sigma_{i j}}{\partial \varepsilon_{k l}^{d}} d \varepsilon_{k l}^{d}$

where $d \varepsilon_{k l}^{m}$ and $d \varepsilon_{k l}^{d}$ come from the decomposition of the total strain increment $d \varepsilon_{i j}=d \varepsilon_{k l}^{m}+d \varepsilon_{k l}^{d}$, referring to the mechanical induced strain increment and the diffusion-induced one, respectively. The mechanical deformation increment is composed of an elastic part $d \varepsilon_{k l}^{e}$ and a plastic part $d \varepsilon_{k l}^{p l}, d \varepsilon_{k l}^{m}=d \varepsilon_{k l}^{e}+d \varepsilon_{k l}^{p l}$, and $\frac{\partial \sigma_{i j}}{\partial \varepsilon_{k l}^{d}}$ is the diffusion-induced modulus tensor which arises due to the nonlinear relationship between the Cauchy stress and the lithium ions' concentration. Such formula has also been adopted by others $[7,13,22]$. In order to update the stress state during the standard interval $\left[t_{n}, t_{n+1}\right]$, we update the total logarithmic strain measure $\left[\varepsilon_{n+1}\right]$ at time $n+1$ by

$\left[\varepsilon_{n+1}\right]=\left[\varepsilon_{n}\right]+[\Delta \bar{\varepsilon}]$

which introduces the rotation-neutralized strain increment $[\Delta \bar{\varepsilon}]$ from time $t_{\mathrm{n}}$ to $t_{\mathrm{n}+1}$. Unless stated otherwise, letters with a top bar are defined in the rotated coordinate. Hughes (1984) used the approximate 2nd order calculation for $[\Delta \bar{\varepsilon}]$ [32], which is given as

$[\boldsymbol{\Delta} \bar{\varepsilon}]=\left[\boldsymbol{R}_{n+1 / 2}\right]^{T}[\Delta \boldsymbol{D}]\left[\boldsymbol{R}_{n+1 / 2}\right]$

where $\Delta \boldsymbol{D}=\operatorname{sym}\left(\frac{\partial \Delta \boldsymbol{u}}{\partial \boldsymbol{x}_{n+1 / 2}}\right)$ is the rotation-neutralized stretch increment tensor relative to the neutralized positions $\boldsymbol{x}_{n+1 / 2}=\frac{1}{2}\left(\boldsymbol{x}_{n}+\boldsymbol{x}_{\mathrm{n}+1}\right)$, and $\left[\boldsymbol{R}_{n+1 / 2}\right]$ comes from the deformation gradient decomposition

$F_{i j}=R_{i k} U_{k j}$

where $R_{i j}$ is the rotation tensor $\left(R_{j i} R_{i j}=\delta_{i j}\right.$ ), and $U_{i j}$ is the right stretch tensor. The updated objective stress defined in the rotated coordinate is given as $\left[\bar{\sigma}_{n+1}\right]=\left[\bar{\sigma}_{n}\right]+[\Delta \bar{\sigma}]$, and the rotated stress increment follows $[\Delta \bar{\sigma}]=[\boldsymbol{C}]\left([\boldsymbol{\Delta} \bar{\varepsilon}]-[\Delta \bar{\varepsilon}]^{p}\right)$, where $[\boldsymbol{C}]$ is the isotropic elastic matrix and $[\Delta \bar{\varepsilon}]^{p}$ is the plastic strain increment. The simple yet powerful method for large inelastic deformation could eliminate the influence of the rigid body rotation on the Cauchy stress, and leads to the same type of elastic-plastic formula for both small deformation and finite deformation cases. The explicit expression of $R_{i j}$ in terms of quaternion parameters is also available [33,34]. One is refereed to Hughes (1984) [32] for detailed algorithm. For force balance, we have

$\frac{\partial \sigma_{i j}}{\partial x_{j}}+f_{i}^{B}=0$

where $\sigma_{i j}$ is the Cauchy stresses, and $f_{i}^{B}$ is the $i$-th component of the body force. The principle of virtual work for mechanical deformation $\delta W_{m}$ is

$\delta W_{m}=\int_{v} \sigma_{i j} \delta D_{i j} d v-\int_{v} f_{i}^{B} \delta u_{i} d v-\int_{s} f_{i}^{s} \delta u_{i} d s=0$

where $f_{i}^{s}$ is the $i$-th component of the surface traction. According to eqn. (9) and eqn. (14), the differentiation of $\delta W_{m}$ is [31].

$d \delta W_{m}=\int_{v} \delta D_{i j} \frac{\partial \sigma_{i j}}{\partial \varepsilon_{k l}^{m}} d D_{k l} d v+\int_{v} \sigma_{i j}\left(\frac{\partial \delta u_{k}}{\partial x_{i}} \frac{\partial d u_{k}}{\partial x_{j}}-2 \delta D_{i k} d D_{k j}\right) d v-$

$\int_{v}\left\{\delta D_{i j}\left(\frac{\partial \sigma_{i j}}{\partial \varepsilon_{k l}^{m}}-\frac{\partial \sigma_{i j}}{\partial \varepsilon_{k l}^{d}}\right) d \varepsilon_{k l}^{d}\right\} d v$

The detailed numerical procedure of the above theory is given in
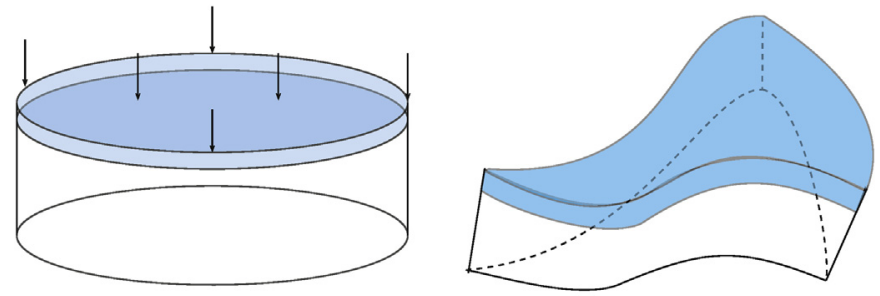

(a)

(b)

Fig. 1. Illustration of a thin film electrode on top of a substrate for curvature measurement. (a) Ions come from the top during the charge/discharge cycle. (b) Deformed structure for stress measurement.

Appendix B, and it has implemented in the commercial finite-element procedure (Abaqus [40]) as a user-element subroutine, which is supplied in the supplementary material. In sec. 3, we apply our theoretical analysis to the charge/discharge process in film-based batteries, and discuss the influence of silicon electrode material with large expansion deformation on the response of film-substrate structure, which is broadly used for in-situ stress measurements.

\section{Stress evolution in the film-substrate structure}

We consider the film-substrate structure shown in Fig. 1, in which an elastic electrode film is adhered to an elastic substrate, which is a typical structure used to reveal the electrode's stress evolution in the charge/discharge process by monitoring the substrate's curvature evolution. The biaxial stress $\sigma_{m}$ is deduced from curvature using the Stoney equation

$\sigma_{m}=\frac{E_{s} h_{s}^{2}}{6\left(1-v_{s}\right) h_{f}} \kappa$

where $E_{s}$ is the elastic modulus of the substrate, $h_{s}$ is the thickness of the substrate, $v_{s}$ is the poisson's ratio of the substrate, and $h_{f}$ is the thickness of the film.

We examine the case when the modulus of electrode materials varies with lithium concentration. Sethuraman et al. (2010) and Hertzberg et al. (2011) reported biaxial moduli of Si thin-film electrode changing with lithium concentration by in-situ measurements and exsitu measurements [16,17]. Shenoy et al. (2010) studied the elastic properties of Li-Si alloys using first-principles calculations for both amorphous and crystalline and found that the modulus linearly decreases with lithium concentration [18], a similar trend was reported in Li-Si alloys [35]. In contrast, the modulus increases with Li concentration for Li-graphite system [36]. Deshpande et al. (2010) showed that the concentration-dependent Young's modulus has a significant effect on stress evolution in the electrodes [9]. It also has a significant effect on in-situ stress measurements. In our simulations, we assume, as did Shenoy et al. (2010) [18], that the elastic modulus, $E_{f}$, varies linearly with the lithium fraction

$E_{f}=E_{0}+\frac{\psi(t)}{1+\psi(t)} m E_{0}$

where $E_{0}$ is the modulus of pure silicon and $m$ is a coefficient.

In mimicking typical battery cells, we consider a film with a radius $R_{f}=5.0 \mu \mathrm{m}$ and a height $h_{f}=0.002 \mu \mathrm{m}$. Its initial elastic modulus and Poisson's ratio are $E_{0}=80 G P a$ and $\nu=0.22$, respectively [37]. The diffusivity coefficient is taken as $D_{0}=1.0 \times 10^{-16} \mathrm{~m}^{2} \mathrm{~s}^{-1}$ [38], and the lithium ions partial molar volume $\Omega=3.497 \times 10^{-6} \mathrm{~m}^{3} \mathrm{~mol}^{-1}$ [11]. For the substrate, its height is $h_{s}=1.0 \mu m$, and the elastic modulus and Poisson's ratio are $E_{s}=162 \mathrm{GPa}, \nu=0.26$, respectively [39]. The top surface of the electrode is in contact with a lithium reservoir. 

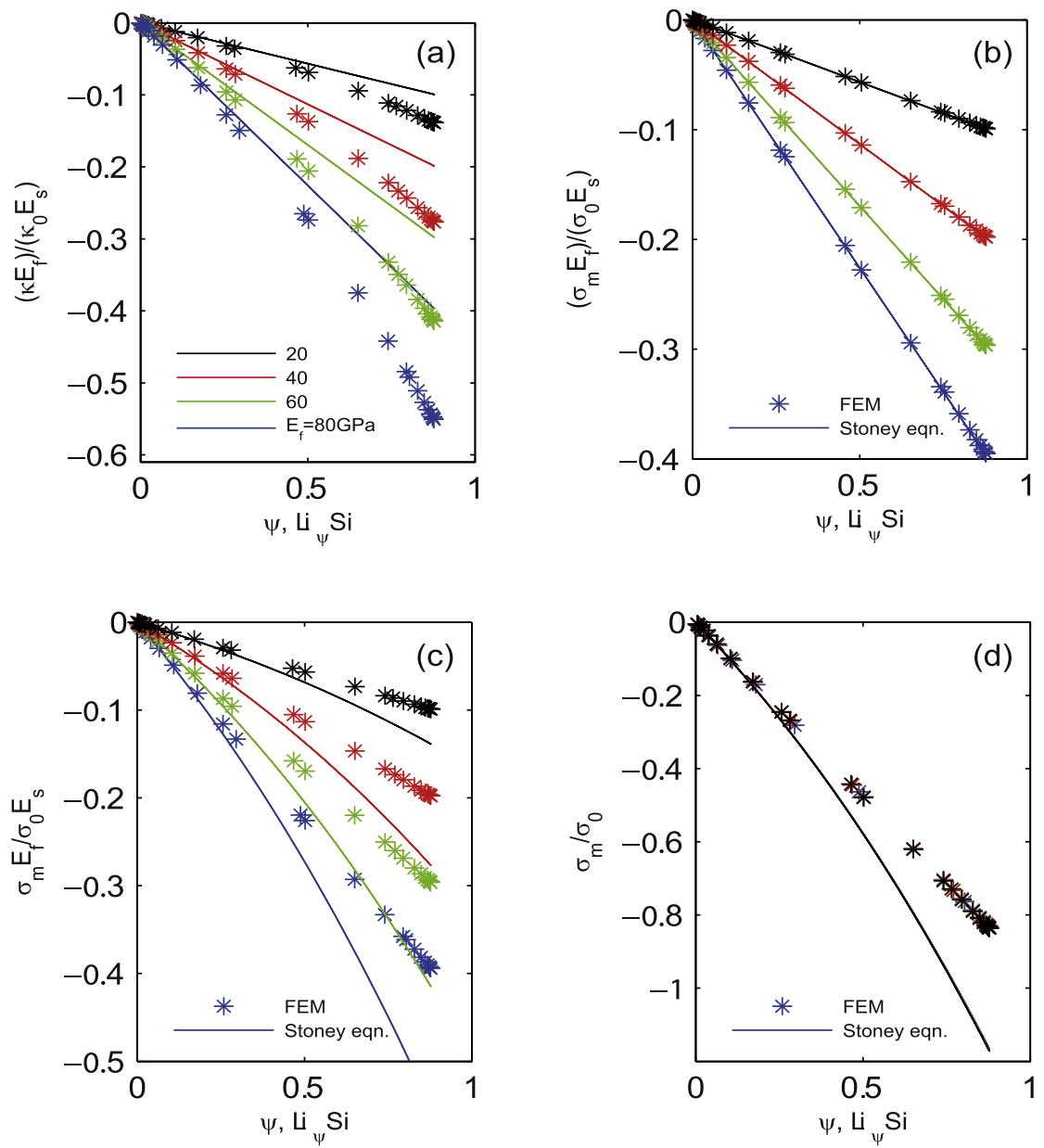

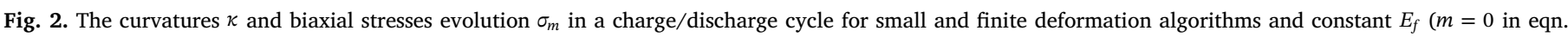

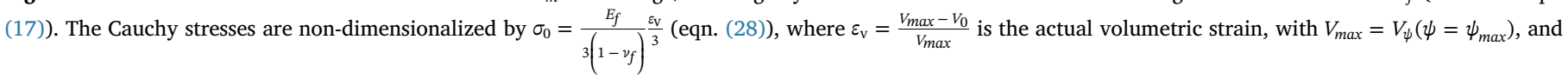

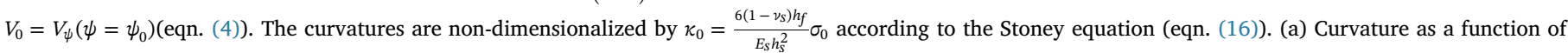

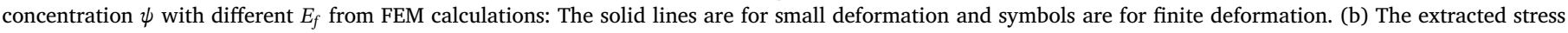

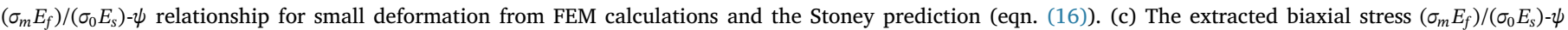
relationship for finite deformation. (d) The extracted biaxial stress $\sigma_{m} / \sigma_{0}-\psi$ relationship for finite deformation.

\subsection{Elastic response in the electrode materials: concentration independent elasticity}

We first examine the case when the electrode layer deforms elastically and the film's elastic modulus $E_{f}$ is independent of concentration, i.e., $m=0$ in eqn. (17). Because the non-dimensional factors $\sigma_{0}$ and $\kappa_{0}$ are linearly increase with the film elastic modulus $E_{f}$, which can eliminate the influence of the elastic modulus on the curvature and the Cauchy stress (show in Fig. 2d), so we multiply $\kappa / \kappa_{0}$ and $\sigma_{m} / \sigma_{0}$ by $E_{f} / E_{s}$ in Fig. 2 and discuss the influence of film's elastic modulus on the curvature and the Cauchy stress. We show in Fig. 2a the curvature variation as a function of the Li fraction. A linear relationship between the curvature and the concentration is seen when the small deformation algorithm is adopted (solid lines) in our FEM calculations. When finite deformation is considered, the obtained curvature- $\psi$ relationship becomes nonlinear even when the material properties are constant during the charge/discharge cycle. The extracted biaxial stress $\sigma_{m}-\psi$ relationship for small deformation from FEM calculations and the Stoney (eqn. (16)) is presented in Fig. 2b. The FEM results are obtained by averaging the bending stress in elements in the electrode layer along the thickness direction, and the Stoney prediction is obtained by knowing the curvature at each $\psi$ from Fig. 2a and substituting it in eqn. (16). For finite deformation, the extracted biaxial stress $\sigma_{m}-\psi$ relationship from FEM calculations deviates significantly from that obtained by the Stoney equation (Fig. 2c). From Fig. 2d, we can see that the non-dimensional quantity $\sigma_{m} / \sigma_{0}$ is independent of the film's elastic modulus $E_{f}$. And the Stoney prediction will break down when the biaxial Cauchy stress is greater than $20 \% \sigma_{0}$. Of course, that occurs when the electrode material deforms elastically; in real battery systems, plastic deformation starts when the stress reaches GPa level.

\subsection{Elastic response in the electrode materials: concentration dependent elasticity}

In this section, we discuss how the concentration dependent modulus affect the stress measurement by FEM calculations based on finite deformation. Fig. 3a presents the curvature-concentration curves for concentration-dependent. We consider the case that higher concentration leads to lower modulus, i.e., $m<0$ in eqn. (17). A weakening in modulus can lower the stress level significantly. Following the same strategy used in Fig. 2, we could extract the biaxial stress $\sigma_{m} / \sigma_{0^{-}} \psi$ curves from both FEM calculations and by using the Stoney equation. As seen from Fig. 3b, the Stoney equation significantly overestimates the stress level in the electrode film, and the Stoney equation can be applied when the biaxial Cauchy stress is lower than $20 \% \sigma_{0}$. 

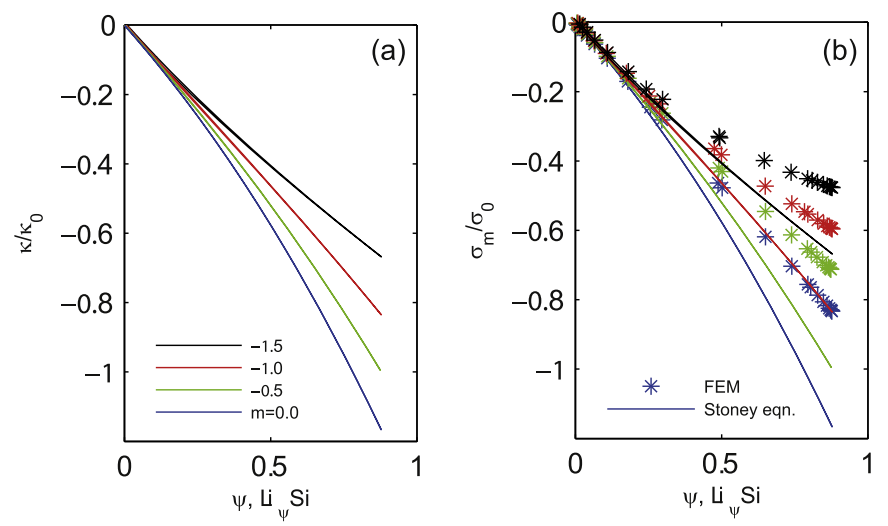

Fig. 3. The influence of electrode materials for the substrate in a charge/discharge cycle, here the electrode materials' elastic modulus varies linearly with the Li fraction (eqn. (17)). (a) The curvature of the substrate from FEM calculations with different $m$. (b) The extracted biaxial stress of the film $\sigma_{m} / \sigma_{0}$ from both FEM calculations (symbols) and prediction by the Stoney equation (eqn. (16)).

\subsection{Geometrical effects}

While the Stoney equation is applicable when the film is thin relative to the substrate, its applicability vs. film thickness has not been firmly established, especially in the context of thin film lithium ion battery electrodes which is the focus of this section.
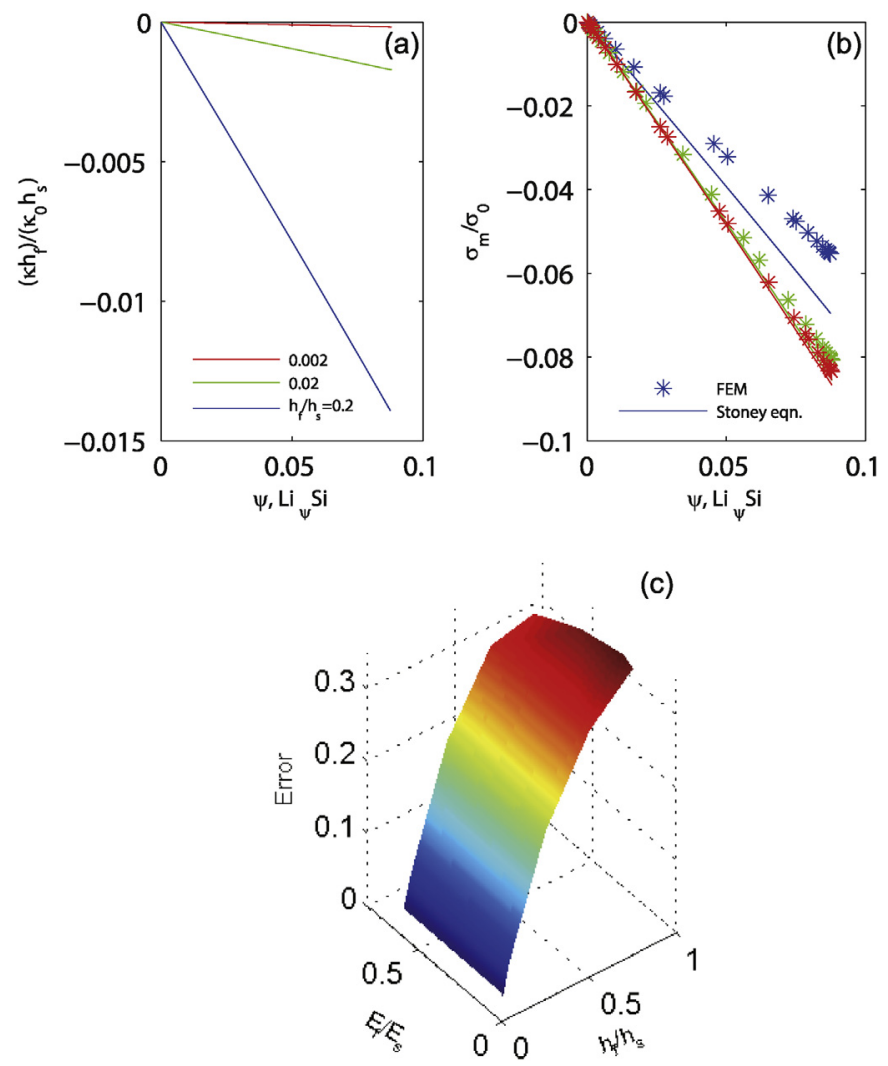

Fig. 4. Effects of the ratio of film to substrate thickness in a charge/discharge cycle. (a) The curvature of the substrate for several thickness ratios, where $h_{f}$ is the film thickness, and $h_{s}$ is the substrate thickness. (b) The biaxial stress evolution of the film where dashed lines are finite deformation FEM analysis results and solid lines are calculated by the Stoney equation and curvature results. (c) The error between the film stress and that acquired from the substrate curvature using the Stoney equation for several $h_{f} / h_{s}$ values, i.e., Error $=\frac{\sigma_{m, \text { stoney }}-\sigma_{m, F E M}}{\sigma_{m, \text { stoney }}}$.
In Fig. 4 a, as in Fig. 2a, we multiply $\kappa / \kappa_{0}$ by $h_{f} / h_{0}$ to eliminate the influence of $\kappa_{0}$, which linearly increases with film's thickness $h_{f}$. We vary the film thickness from $h_{f}=0.002 \mu m$ to $0.2 \mu m$. Fig. 4a shows the curvature- $\psi$ curves for different $h_{f} / h_{s}$. Corresponding $\sigma_{m} / \sigma_{0}-\psi$ curves are presented in Fig. 4b. The difference between the FEM calculations and the Stoney equation prediction is apparent when $\frac{h_{f}}{h_{s}}>0.02$, and the stress predicted by the latter is greater than the FEM results. We constructed the stress error map for Stoney equation predictions as a function of the thickness ratio $h_{f} / h_{s}$ and presented it in Fig. 4c. We see that the Young's modulus of the electrode material may also influence the prediction but its influence is weaker than the thickness ratio.

\subsection{Rigid plastic electrode}

We now explore more complicated electrode material constitutive behavior by taking plasticity into consideration. We show in Fig. 5a the stress-strain curves of the electrode material with different constitutive behavior. Both equivalent stress $\sigma_{e}$ and equivalent strain $\varepsilon_{e}$ are used, with $\sigma_{e}=\sqrt{\frac{3}{2}\left(\sigma_{i j}-\frac{1}{3} \sigma_{i j} \delta_{i j}\right)\left(\sigma_{i j}-\frac{1}{3} \sigma_{i j} \delta_{i j}\right)}$ and $\varepsilon_{e}=\sqrt{\frac{2}{3}\left(\varepsilon_{i j}-\frac{1}{3} \varepsilon_{i j} \delta_{i j}\right)\left(\varepsilon_{i j}-\frac{1}{3} \varepsilon_{i j} \delta_{i j}\right)}$, the Kronecker delta $\delta_{i j}=1$ if $i=j$, otherwise $\delta_{i j}=0$. We consider the material to be elastic-perfectly plasticity. For case I, the Young's modulus $E_{f}$ is a constant, i.e., $m=0$ in eqn. (17); and for case II, $E_{f}$ decreases with increasing lithium concentration. We use $m=-0.79$ as recommended by Shenoy et al. (2010) [18]. From calculated material response shown in Fig. 5a, we see that the concentration-dependent Young's modulus would result in an apparent difference in the elastic deformation stage when charge induced strain is large (high concentration). Modulus value from the unloading curve for case II is smaller than that of case I. In Fig. 5b, we present the curvature evolution within a full lithiation/delithiation cycle from our FEM simulation. Lithiation induced elasticity softening leads to smaller curvature during the delithiation stage, in contrast to electrode with constant modulus. In Fig. 5c, we show the extracted biaxial stress vs. $\psi$ from FEM calculations and that based on Stoney prediction (eqn. (16)). The blue and red curves, for the respective case I and II, are predicted by FEM calculations. They showed the Cauchy stress evolution during lithiation/delithiation. Corresponding predictions by the Stoney equation (based on eqn. (16) and the curvature curve in Fig. 5b) are shown in green and black. Stoney equation cannot accurately predict the biaxial stress in the plastic deformation stage. For case I, the error originates from the fact that Stoney equation was derived for small deformation while the electrode material experiences large deformation; the additional concentration-dependent elasticity in case II complicates the situation which further challenges the applicability of Stoney equation.

\section{Discussions and conclusions}

For better understanding the stress evolution in electrodes during lithiation and delithiation, there is a compelling need for more robust numerical methods to solve the electrochemical-mechanical coupled problems. In this paper, we presented a diffusion-mechanics coupling constitutive model and developed a robust numerical method to solve the coupled fields during finite deformation. The numerical method is also presented in detail in the supplementary material. We characterize the influences of finite-deformation, concentration-dependent Young's modulus, as well as film-substrate thickness ratio on the beam stress in electrode materials. These effects are normally neglected in stressmeasurement using the Stoney equation. We conclude that a complementary fully coupled numerical simulation should be used, in combination with experimental measurements, to extract the actual mechanical response and critical material parameters by in-situ curvature measurements. 

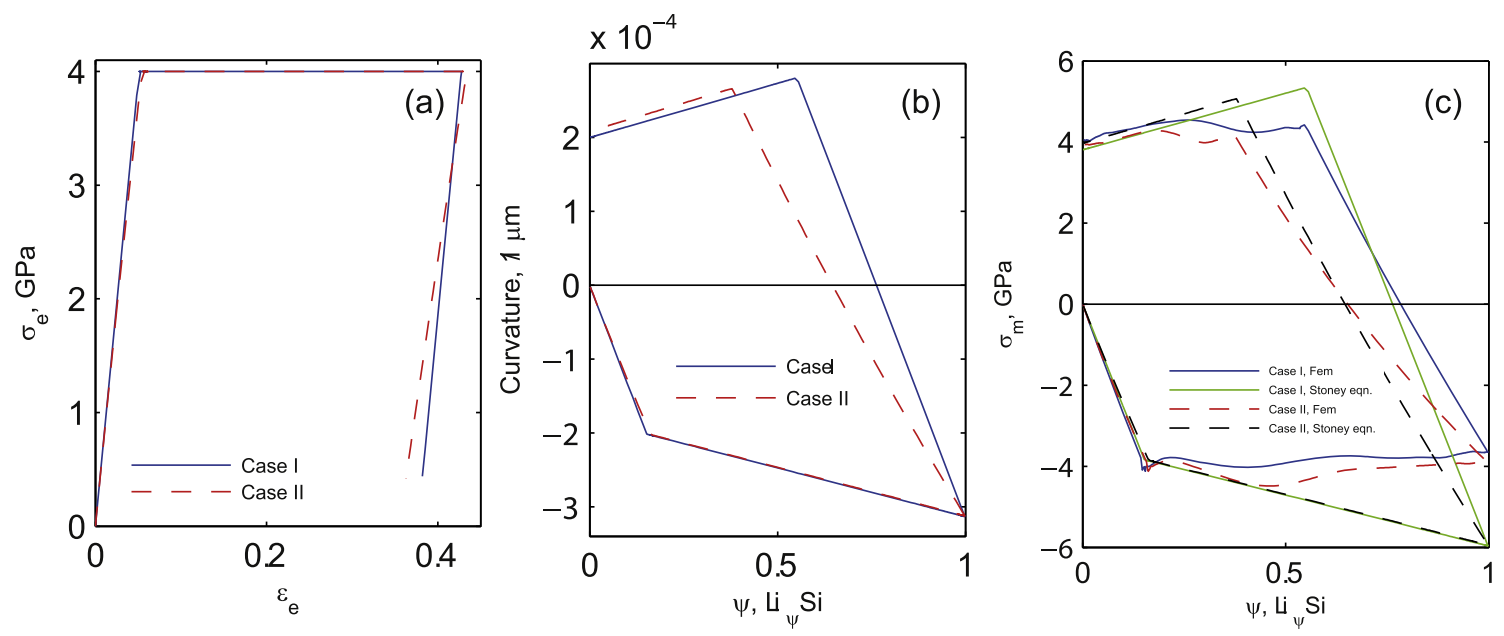

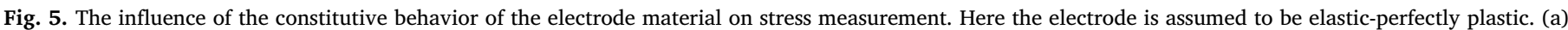

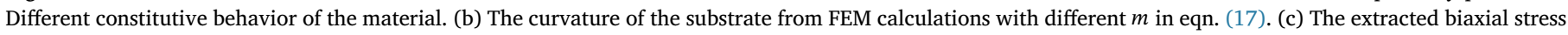
vs. $\psi$ from FEM calculations and that based on Stoney equation prediction (eqn. (16)).

\section{Acknowledgments}

The authors acknowledge support from the National Natural Science Foundation of China (NSFC) (Grant no. 11425211), the Strategic
Priority Research Program of the Chinese Academy of Sciences (XDB22020200). YTC would like to thank US National Science Foundation award 1355438 "Powering the Kentucky Bioeconomy for a Sustainable Future."

\section{Appendix A. Stress-curvature relation in films with linear-elastic response during charge-discharge}

Now we examine the case when the modulus of the electrode layer is concentration dependent with linear-elastic response. We aim to give the corresponding simplified curvature-stress (or concentration) relationship. The biaxial stress of the electrode film $\sigma_{m}$ induced by the mismatch strain $\varepsilon_{m}$ is given for linear elastic electrode materials as

$\sigma_{m}=\frac{E}{1-v_{f}} \varepsilon_{m}$

where $E$ and $\nu_{f}$ are elastic modulus and Poisson's radio, and the mismatch strain $\varepsilon_{m}$ is related to the concentration $c(t)$ by

$\varepsilon_{m}=\alpha\left(c(t)-c_{0}\right)$

where $c_{0}$ is the initial concentration in the electrode materials. The film's capacity is

$\mathrm{Ca}=\frac{i t}{\rho_{f} h_{f}}$

where $i$ is the current density on the electrode film, $t$ is time, $\rho_{f}$ is the density of the electrode material, $h_{f}$ is the thickness of the electrode. And the slope of the stress-capacity curve is

$K_{s c}=\frac{d \sigma_{m}}{d C a}$

Thus, the slope of the stress-capacity curve eqn. (21) is given according to eqn. (18)-(20) as

$K_{s c}=\frac{\rho_{f} h_{f}}{i} \frac{\alpha}{1-v_{f}} \frac{\partial\left(E\left(c(t)-c_{0}\right)\right)}{\partial\left(c(t)-c_{0}\right)} \frac{d c(t)}{d t}$

Because the height of the electrode film is small, $R \gg h_{f}$. We may assume one dimensional diffusion along the thickness direction [21],

$\frac{\partial c}{\partial t}=D \frac{\partial^{2} c}{\partial h^{2}}$

with boundary conditions and initial conditions given below [23].

$\left.\frac{\partial c}{\partial t}\right|_{h=0}=0$

$\left.D \frac{\partial c}{\partial h}\right|_{h=h f}=\frac{i}{F}$

$c(h, 0)=c_{0}$

Hence the concentration as a function of time and thickness is given as 
$c(h, t)=c_{0}-\frac{i h_{f}}{6 D F}+\frac{i}{2 D F h_{f}} h^{2}+\frac{i t}{h_{f} F}+\sum_{n=1,3,5}^{\infty} \frac{4 i h_{f}}{n^{2} \pi^{2} D F} \cos \frac{n \pi h}{h_{f}} e^{-\frac{n^{2} \pi^{2}}{h_{f}^{2}} D t}$

The average concentration in the film is

$c_{a v}=\frac{\int_{0}^{h_{f}} c(h, t) d h}{h_{f}}=\frac{i t}{F h_{f}}$

According to eqn. (22) and eqn. (26), the slope of the film due to lithiation is

$K_{s c}=\frac{\alpha \rho_{f}}{\left(1-v_{f}\right) F} \frac{\partial\left(E\left(c(t)-c_{0}\right)\right)}{\partial\left(c(t)-c_{0}\right)}$

According to eqn. (27), when the elastic modulus is independent of ion concentration, $K_{s c}=\frac{E_{0} \alpha_{f}}{\left(1-v_{f}\right) F}$ is a constant. Otherwise, according to eqn. (27), the biaxial stress is given as

$\sigma_{m}=\sigma_{0}-\int_{0}^{C a(t)} K_{s c} d C a=\sigma_{0}-\frac{\alpha}{\left(1-v_{f}\right)} E\left(c(t)-c_{0}\right)$

where $\sigma_{0}$ is the initial residual stress in the electrode film. It is noted that the accuracy of eqn. (28) is restricted to films subjected to small deformation.

\section{Appendix B. Numerical procedure}

In this appendix, we provide the detailed numerical procedures to implement the electrochemical-mechanical coupling differential equations. The variation of Eqn. (5a) is given as

$\delta W_{d}=\int_{v} \delta \psi H(\psi) \frac{\partial \psi}{\partial t} d v-\int_{v} \delta \psi_{, i} J_{i} d v-\int_{s} J^{s} \delta \psi d s=0$

where $\delta \psi$ is the admissible virtual normalized concentration. The weak form of the force balance equation in eqn. (13) is then written as

$\delta W_{m}=\int_{v} \delta D_{i j} \sigma_{i j} d v-\int_{v} \delta u_{i} f_{i}^{B} d v-\int_{s} \delta u_{i} f_{i}^{s} d s=0$

where $\delta D_{i j}$ denotes the virtual strain. In order to solve the integral form of Eqn. (29) and Eqn. (30), we discretize the concentration field and the displacement field. For a typical element with $M$ nodes, we denote the $i$-th coordinate of the $a$-th node $x_{i}^{a}$, where the superscript $a$ ranges from 1 to $M$. The normalized concentration $\psi\left(x_{i}\right)$ and the displacement $u_{i}\left(x_{i}\right)$ are interpolated as

$\psi\left(x_{i}\right)=\sum_{a=1}^{M} N^{a}\left(x_{i}\right) \psi^{a}, \quad u_{i}\left(x_{i}\right)=\sum_{a=1}^{M} N^{a}\left(x_{i}\right) u_{i}^{a}$

where $N^{a}\left(x_{i}\right)$ are the shape functions. Time integration in transient problems utilizes the backward Euler method: $\frac{\partial \psi}{\partial t}=\frac{\psi_{t+\Delta t}-\psi_{t}}{\Delta t}$. Then the coupled equations are solved using a Newton-Raphson iteration procedure by defining the following element-level residuals for the normalized concentration and the displacement,

$R_{\psi}^{a}=-\frac{\partial \delta W_{d}}{\partial \delta \psi^{a}}=-\int_{v} N^{a} \frac{\phi_{t+\Delta t}-\phi_{t}}{\Delta t} d v+\int_{v} \frac{\partial N^{a}}{\partial x_{i}} J_{i} d v+\int_{s} N^{a} J^{s} d s=0$

$R_{u_{p}}^{a}=-\frac{\partial \delta W_{m}}{\partial \delta u_{p}^{a}}=-\int_{v}\left(\frac{\partial N^{a}}{\partial x_{i}} \delta_{j p}+\frac{\partial N^{a}}{\partial x_{j}} \delta_{i p}\right) \sigma_{i j} d v+\int_{v} N^{a} f_{p}^{B} d v+\int_{s} N^{a} f_{p}^{s} d s=0$

Taking the two dimensional problem as an example, the overall elemental residuals in matrix form are given by

$R=\left[\begin{array}{llllllllll}R_{u_{1}}^{1} & R_{u_{2}}^{1} & R_{\psi}^{1} & R_{u_{1}}^{2} & R_{u_{2}}^{2} & R_{\psi}^{2} & \cdots & R_{u_{1}}^{M} & R_{u_{2}}^{M} & R_{\psi}^{M}\end{array}\right]^{T}$

In addition to the residuals, the following Jacobian matrix is also required for the iterative Newton-Raphson method, which is defined as

$K_{\psi \psi}^{a b}=-\frac{\partial R_{\psi}^{a}}{\partial \psi^{b}}=\int_{v} N^{a} H\left(\psi_{t+\Delta t}\right) \frac{N^{b}}{\Delta t} d v+\int_{v} \frac{\partial N^{a}}{\partial x_{i}} D_{0} H\left(\psi_{t+\Delta t}\right) \frac{\partial N^{b}}{\partial x_{i}} d v-\int_{v} \frac{\partial N^{a}}{\partial x_{i}} \frac{D_{0} \Omega}{R T} \sigma_{h, i} H\left(\psi_{t+\Delta t}\right) N^{b} d v$

and

$K_{\psi u_{q}}^{a b}=-\frac{\partial R_{\psi}^{a}}{\partial u_{q}^{b}}=-\int_{v}\left[\frac{\partial N^{a}}{\partial x_{i}} G_{, q}\left(\psi_{t+\Delta t}\right) \frac{\partial N^{b}}{\partial x_{i}}+\frac{\partial N^{a}}{\partial x_{q}} G_{, i}\left(\psi_{t+\Delta t}\right) \frac{\partial N^{b}}{\partial x_{i}}\right] d v$ 


$$
\begin{aligned}
K_{u_{p} u_{q}}^{a b}= & -\frac{\partial R_{u_{p}}^{a}}{\partial u_{l}^{b}}=\int_{v}\left(\frac{\partial N^{a}}{\partial x_{i}} \delta_{j p}+\frac{\partial N^{a}}{\partial x_{j}} \delta_{i p}\right) \frac{\partial \sigma_{i j}^{J}}{\partial \varepsilon_{k l}^{m}}\left(\frac{\partial N^{b}}{\partial x_{k}} \delta_{l q}+\frac{\partial N^{b}}{\partial x_{l}} \delta_{k q}\right) d v \\
& +\int_{v} \sigma_{i j}\left[\frac{\partial N^{a}}{\partial x_{i}} \frac{\partial N^{b}}{\partial x_{j}} \delta_{p q}-2\left(\frac{\partial N^{a}}{\partial x_{i}} \delta_{n p}+\frac{\partial N^{a}}{\partial x_{n}} \delta_{i p}\right)\left(\frac{\partial N^{b}}{\partial x_{n}} \delta_{j q}+\frac{\partial N^{b}}{\partial x_{j}} \delta_{n q}\right)\right] d v \\
K_{u_{p} \psi}^{a b}= & -\frac{\partial R_{u_{p}}^{a}}{\partial \psi^{b}}=-\int_{v}\left[\left(\frac{\partial N^{a}}{\partial x_{i}} \delta_{j p}+\frac{\partial N^{a}}{\partial x_{j}} \delta_{i p}\right)\left(\frac{\partial \sigma_{i j}}{\partial \varepsilon_{k l}^{m}}-\frac{\partial \sigma_{i j}}{\partial \varepsilon_{k l}^{d}}\right) \alpha \delta_{k l} N^{b}\right] d v
\end{aligned}
$$

For brevity, we assemble the Jacobian matrix of two dimensions elements as

$$
K=\left[\begin{array}{cccccccccc}
K_{u_{1} u_{1}}^{11} & K_{u_{1} u_{2}}^{11} & K_{u_{1} \psi}^{11} & K_{u_{1} u_{1}}^{12} & K_{u_{1} u_{2}}^{12} & K_{u_{1} \psi}^{12} & & K_{u_{1} u_{1}}^{1 M} & K_{u_{1} u_{2}}^{1 M} & K_{u_{1} \psi}^{1 M} \\
K_{u_{2} u_{1}}^{11} & K_{u_{2} u_{2}}^{11} & K_{u_{2} \psi}^{11} & K_{u_{2} u_{1}}^{12} & K_{u_{2} u_{2}}^{12} & K_{u_{2} \psi}^{12} & & K_{u_{2} u_{1}}^{1 M} & K_{u_{2} u_{2}}^{1 M} & K_{u_{2} \psi}^{1 M} \\
K_{\psi u_{1}}^{11} & K_{\psi u_{2}}^{11} & K_{\psi \psi}^{11} & K_{\psi u_{1}}^{12} & K_{\psi u_{2}}^{12} & K_{\psi \psi}^{12} & \ldots & K_{\psi u_{1}}^{1 M} & K_{\psi u_{2}}^{1 M} & K_{\psi \psi}^{1 M} \\
K_{u_{1} u_{1}}^{21} & K_{u_{1} u_{2}}^{21} & K_{u_{1} \psi}^{21} & K_{u_{1} u_{1}}^{22} & K_{u_{1} u_{2}}^{22} & K_{u_{1} \psi}^{22} & & K_{u_{1} u_{1}}^{2 M} & K_{u_{1} u_{2}}^{2 M} & K_{u_{1} \psi}^{2 M} \\
K_{u_{2} u_{1}}^{21} & K_{u_{2} u_{2}}^{21} & K_{u_{2} \psi}^{21} & K_{u_{2} u_{1}}^{22} & K_{u_{2} u_{2}}^{22} & K_{u_{2} \psi}^{22} & & K_{u_{2} u_{1}}^{2 M} & K_{u_{2} u_{2}}^{2 M} & K_{u_{2} \psi}^{2 M} \\
K_{\psi u_{1}}^{121} & K_{\psi u_{2}}^{21} & K_{\psi \psi}^{21} & K_{\psi u_{1}}^{22} & K_{\psi u_{2}}^{22} & K_{\psi \psi}^{22} & & K_{\psi u_{1}}^{2 M} & K_{\psi u_{2}}^{2 M} & K_{\psi \psi}^{2 M} \\
& & \vdots & & & & \ddots & & \vdots & \\
K_{u_{1} u_{1}}^{M 1} & K_{u_{1} u_{2}}^{M 1} & K_{u_{1} \psi}^{M 1} & K_{u_{1} u_{1}}^{M 2} & K_{u_{1} u_{2}}^{M 2} & K_{u_{1} \psi}^{M 2} & & K_{u_{1} u_{1}}^{M M} & K_{u_{1} u_{2}}^{M M} & K_{u_{1} \psi}^{M M} \\
K_{u_{2} u_{1}}^{M 1} & K_{u_{2} u_{2}}^{M 1} & K_{u_{2} \psi}^{M 1} & K_{u_{2} u_{1}}^{M 2} & K_{u_{2} u_{2}}^{M 2} & K_{u_{2} \psi}^{M 2} & \ldots & K_{u_{2} u_{1}}^{M M} & K_{u_{2} u_{2}}^{M M} & K_{u_{2} \psi}^{M M} \\
K_{\psi u_{1}}^{M 1} & K_{\psi u_{1}}^{M 1} & K_{\psi \psi}^{M 1} & K_{\psi u_{1}}^{M 2} & K_{\psi u_{2}}^{M 2} & K_{\psi \psi}^{M 2} & & K_{\psi u_{1}}^{M M} & K_{\psi u_{2}}^{M M} & K_{\psi \psi}^{M M}
\end{array}\right]
$$

The residuals (eqn. (32)) and the Jacobian matrix (eqn. (34)) are implemented in the commercial FEM package (Abaqus [40]) by developing a user-element subroutine, which is supplied in the supplementary material.

\section{Appendix C. Supplementary data}

Supplementary data related to this article can be found at http://dx.doi.org/10.1016/j.jpowsour.2018.03.052

\section{References}

[1] A.S. Aricò, P. Bruce, B. Scrosati, J.M. Tarascon, W. van Schalkwijk, Nanostructured materials for advanced energy conversion and storage devices, Nat. Mater. 4 (2005) 366-377.

[2] M. Armand, J.M. Tarascon, Building better batteries, Nature 451 (2008) 652-657.

[3] J.W. Long, B. Dunn, D.R. Rolison, H.S. White, Three-dimensional battery architectures, Chem. Rev. 104 (2004) 4463-4492.

[4] M.N. Obrovac, L. Christensen, Structural changes in silicon anodes during lithium insertion/extraction, Electrochem. Solid State Lett. 7 (2004) A93-A96.

[5] T.D. Hatchard, J.R. Dahn, In situ XRD and electrochemical study of the reaction of lithium with amorphous silicon, J. Electrochem. Soc. 151 (2004) A838-A842.

[6] A.F. Bower, P.R. Guduru, E. Chason, Analytical solutions for composition and stress in spherical elastic-plastic lithium-ion electrode particles containing a propagating phase boundary, Int. J. Solid Struct. 69 (2015) 328-342.

[7] A.F. Bower, P.R. Guduru, V.A. Sethuraman, A finite strain model of stress, diffusion, plastic flow and electrochemical reactions in a lithium-ion half-cell, J. Mech. Phys. Solid. 59 (2011) 804-828.

[8] J. Christensen, J. Newman, Stress generation and fracture in lithium insertion materials, J. Solid State Electrochem. 10 (2006) 293-319.

[9] R. Deshpande, Y. Qi, Y.T. Cheng, Effects of concentration-dependent elastic modulus on diffusion-induced stresses for battery applications, J. Electrochem. Soc. 157 (2010) A967-A971.

[10] C.V. Di Leo, E. Rejovitzky, L. Anand, A Cahn-Hilliard-type phase-field theory for species diffusion coupled with large elastic deformations: application to phase-separating Li-ion electrode materials, J. Mech. Phys. Solid. 70 (2014) 1-29.

[11] X. Zhang, W. Shyy, A.M. Sastry, Numerical simulation of intercalation-induced stress in Li-ion battery electrode particles, J. Electrochem. Soc. 154 (2007) A910-A916.

[12] D.R. Baker, M.W. Verbrugge, A.F. Bower, Swelling and elastic deformation of lithium-silicon electrode materials, J. Electrochem. Soc. 163 (2016) A624-A631.

[13] L. Anand, A Cahn-Hilliard-type theory for species diffusion coupled with large elastic -plastic deformations, J. Mech. Phys. Solid. 60 (2012) 1983-2002.

[14] H. Xie, Q. Zhang, H. Song, B. Shi, Y. Kang, Modeling and in situ characterization of lithiation-induced stress in electrodes during the coupled mechano-electro-chemical process, J. Power Sources 342 (2017) 896-903.
[15] G.G. Stoney, The tension of metallic films deposited by electrolysis. Proceedings of the Royal Society of London, Series A, Containing Papers of a Math. and Phys. Character 82 (1909) 172-175.

[16] B. Hertzberg, J. Benson, G. Yushin, Ex-situ depth-sensing indentation measurements of electrochemically produced Si-Li alloy films, Electrochem. Commun. 13 (2011) $818-821$

[17] V.A. Sethuraman, M.J. Chon, M. Shimshak, N. Van Winkle, P.R. Guduru, In situ measurement of biaxial modulus of Si anode for Li-ion batteries, Electrochem. Commun. 12 (2010) 1614-1617.

[18] V.B. Shenoy, P. Johari, Y. Qi, Elastic softening of amorphous and crystalline Li-Si phases with increasing Li concentration: a first-principles study, J. Power Sources 195 (2010) 6825-6830.

[19] S.K. Soni, B.W. Sheldon, X. Xiao, A.F. Bower, M.W. Verbrugge, Diffusion mediated lithiation stresses in Si thin film electrodes, J. Electrochem. Soc. 159 (2012) $1520-1527$.

[20] S.K. Soni, B.W. Sheldon, X. Xiao, M.W. Verbrugge, A. Dongjoon, H. Haftbaradaran, G. Huajian, Stress mitigation during the lithiation of patterned amorphous Si islands, J. Electrochem. Soc. 159 (2011) 38-43.

[21] H.S. Carslaw, J.C. Jaeger, H. Feshbach, Conduction of heat in solids, Phys. Today 15 (1962) 74-76.

[22] S.A. Chester, C.V. Di Leo, L. Anand, A finite element implementation of a coupled diffusion-deformation theory for elastomeric gels, Int. J. Solid Struct. 52 (2015) $1-18$.

[23] Y.T. Cheng, M.W. Verbrugge, Evolution of stress within a spherical insertion electrode particle under potentiostatic and galvanostatic operation, J. Power Sources 190 (2009) 453-460.

[24] J.C.M. Li, Physical chemistry of some microstructural phenomena, Metallurgical Transactions A 9 (1978) 1353-1380.

[25] Y. Wei, A.F. Bower, H. Gao, Analytical model and molecular dynamics simulations of the size dependence of flow stress in amorphous intermetallic nanowires at temperatures near the glass transition, Phys. Rev. B 81 (2010) 125402.

[26] F. Yang, Interaction between diffusion and chemical stresses, Mater. Sci. Eng. 409 (2005) 153-159.

[27] X. Zhang, A.M. Sastry, W. Shyy, Intercalation-induced stress and heat generation within single lithium-ion battery cathode particles, J. Electrochem. Soc. 155 (2008) A542-A552.

[28] F. Fan, S. Huang, H. Yang, M. Raju, D. Datta, V.B. Shenoy, A.C.T. Duin, S. Zhang, 
T. Zhu, Mechanical properties of amorphous LixSi alloys: a reactive force field study, Modelling and Simulation in Materials Science and Engineering 21 (2013) 074002.

[29] Y. He, X. Yu, G. Li, R. Wang, H. Li, Y. Wang, H. Gau, X. Huang, Shape evolution of patterned amorphous and polycrystalline silicon microarray thin film electrodes caused by lithium insertion and extraction, J. Power Sources 216 (2012) 131-138.

[30] B.A. Boukamp, G.C. Lesh, R.A. Huggins, All-solid lithium electrodes with mixedconductor matrix, J. Electrochem. Soc. 128 (1981) 725-729.

[31] R.M. McMeeking, J.R. Rice, Finite-element formulations for problems of large elastic-plastic deformation, Int. J. Solid Struct. 11 (1975) 601-616.

[32] T.J.R. Hughes, Numerical Implementation of Constitutive Models: Rate-independent Deviatoric Plasticity. Theoretical Foundation for Large-scale

Computations for Nonlinear Material Behavior, Springer Netherlands, 1984, pp. 29-63.

[33] M.A. Crisfield, Non-linear Finite Element Analysis of Solids and Structures vol. 2,
John Wiley\&Sons, Chichester, 1997 Advanced Topic.

[34] T.J.R. Hughes, J.C. Simo, Computational Inelasticity, (1998) New York.

[35] M.E. Stournara, P.R. Guduru, V.B. Shenoy, Elastic behavior of crystalline Li-Sn phases with increasing Li concentration, J. of Power Sour 208 (2012) 165-169.

[36] Y. Qi, H. Guo, L.G. Hector, A. Timmons, Threefold increase in the Young's modulus of graphite negative electrode during lithium intercalation, J. Electrochem. Soc. 157 (2010) A558-A566.

[37] L.B. Freund, S. Suresh, Thin Film Materials: Stress, Defect Formation and Surface Evolution, Cambridge University Press UK, 2004.

[38] J. Pan, Q. Zhang, J. Li, M.J. Beck, X. Xiao, Y.T. Cheng, Effects of stress on lithium transport in amorphous silicon electrodes for lithium-ion batteries, Nanomater. Energy 13 (2015) 192-199.

[39] W.A. Brantley, Calculated elastic constants for stress problems associated with semiconductor devices, J. Appl. Phys. 44 (1973) 534-535.

[40] ABAQUS/Standard, SIMULIA, Providence, RI, 2013. 